\title{
Creciente desarrollo del sector lechero en China
}

\section{DOI: $10.32870 /$ mycp.v7i22.631}

\section{I}

\section{ntroducción}

En China, no obstante los prejuicios sobre el consumo de leche principalmente en zonas rurales, en los últimos años, particularmente desde los años noventa, se han mostrado crecientes niveles de consumo de leche de vaca, así como de sus productos derivados (leche en polvo, suero, yogurt, queso y mantequilla, entre otros). Dicho crecimiento en el consumo se debe a un creciente nivel de ingresos y a una mayor difusión en los medios, los cuales resaltan los beneficios del consumo adecuado de leche. Lo anterior es relevante debido a que, por el tamaño de la población de China, un incremento en su consumo de lácteos, podría provocar un incremento importante en los precios internacionales de la leche, lo cual no es bueno para países que son importadores netos de leche, como es el caso de México.

\section{Producción}

En China se producen casi de todas las leches que se consumen en el mundo, como la de búfala, cabra, camello, vaca y oveja. Aunque China ocupa el primer lugar de producción mundial de leche de oveja, es en la de vaca donde representa internamente una mayor proporción y donde mejor dinamismo en el crecimiento ha mostrado. En los últimos trece años, de 1990 a 2003 , se registró un crecimiento en la producción de leche de vaca de $228 \%$, al pasar de una producción de 4362 millones de litros en 1990 a 14335 en 2003; en la de oveja el crecimiento fue de $70 \%$, y en la de búfala y de cabra, de poco más de $40 \%$. En esos años también cambió la proporción de leche bovina con respecto al total de la producción de leches, ya que de representar $62 \%$ en 1990 , pasó a $78 \%$ en 2003 (ver cuadro anexo).

* Investigador del Departamento de Estudios del Pacífico, de la Universidad de Guadalajara.

\author{
Ramón Robledo Padilla*
}


embargo, el tamaño de la población de China provoca que tan sólo el incremento de un litro en el consumo per cápita de leche represente una demanda de casi 1300 millones de litros más, por lo que hacen de este mercado un atractivo potencial muy importante para los países exportadores de lácteos en los años que están por venir.

Cuando los países son importadores netos $^{3}$ (el caso de México), el incremento en el consumo de lácteos en China no es tan alentador debido a que un incremento acelerado en la demanda mundial de estos productos provocaría un aumento en los precios internacionales y, por consecuencia, en nuestro país, una mayor salida de divisas por concepto de importaciones.

\section{Consumo}

El consumo de leche en China se ha incrementado de manera importante en los últimos 20 años, pero comparado con otros países, su consumo per cápita continúa siendo bajo. Por ejemplo, donde más se consume leche es en los países desarrollados como Canadá, Estados Unidos y la mayoría de Europa; en cambio en Asia el consumo de leche es mucho más bajo. En 2001, mientras que en los primeros el consumo fue mayor a 200 litros per cápita anualmente, en China se consumieron poco más de nueve litros. ${ }^{4}$ Si contamos las otras leches producidas en China, como la de búfala, oveja, cabra y camella, el consumo per cápita fue 12.7 litros. ${ }^{5}$

Históricamente, el hábito de ingerir leche y sus derivados ha sido muy escaso en China debido a varias razones: el índice elevado de intolerancia a la lactosa (casi $80 \%$ de la población presenta este síntoma a la edad de ocho años ${ }^{6}$; el bajo nivel de producción de leche comparado con el tamaño de su población; inconvenientes como el clima, la falta de transporte y de almacenes adecuados. Sin embargo, desde hace 25 años, particularmente durante la última década del siglo XX, los patrones de consumo en China han ido cambiando, ${ }^{7}$ en especial en las áreas urbanas, donde los ingresos de la población en promedio son mayores.

En estos lugares, siguiendo la tendencia de los hábitos de consumo de los países ricos, conforme se incrementan los ingresos, cada vez se consumen más los productos de origen animal, como la carne de carnero, cerdo, res y ave, además del huevo y la leche. Otra característica importante es que los lácteos en China, a excepción de los otros productos mencionados, presentan características de un bien de lujo, debido a que tiene una elasticidad-ingreso de la demanda de $1.26,{ }^{8}$ es decir, mayor que uno; en cambio, los otros cinco bienes están dentro de los considerados como necesarios porque sus elasticidades son menores a uno. ${ }^{9}$ Esto quiere decir que para el caso de los lácteos

Cuadro 1

Indicadores de la leche en China (millones de litros)

\begin{tabular}{ccccccc}
\hline Año & Producción & Importación & Existencias & Exportaciones & Consumo & Autosuficiencia en leche \\
\hline 1990 & 7,037 & 1,211 & & 102 & 8,146 & 86.39 \\
1991 & 7,603 & 1,376 & 2 & 146 & 8,837 & 86.04 \\
1992 & 8,072 & 1,350 & & 177 & 9,245 & 87.31 \\
1993 & 8,150 & 1,434 & -4 & 231 & 9,349 & 87.18 \\
1994 & 8,682 & 2,052 & 0 & 517 & 10,216 & 84.98 \\
1995 & 9,458 & 1,843 & 4 & 502 & 10,802 & 87.56 \\
1996 & 10,191 & 1,743 & & 431 & 11,504 & 88.59 \\
1997 & 10,094 & 1,923 & -4 & 519 & 11,495 & 87.81 \\
1998 & 10,517 & 1,867 & -2 & 526 & 11,855 & 88.71 \\
1999 & 11,244 & 2,073 & -4 & 451 & 12,863 & 87.41 \\
2000 & 12,374 & 2,110 & 5 & 546 & 13,943 & 88.75 \\
2001 & 14,490 & 1,900 & 5 & 371 & 16,024 & 90.43 \\
\hline
\end{tabular}

Fuente: http://www.fao.org. 
en China, a medida que el ingreso se incremente, habrá un aumento en la demanda de estos productos, proporcionalmente mayor. ${ }^{10}$

En general, la principal fuente de calorías y proteínas en la dieta de los chinos continúa basándose en cereales, verduras y frutas; asimismo, la carne de puerco es la preferida en la dieta de los chinos. No obstante, como ya se mencionó, aunque los chinos cada vez consumen más leche, no es significativo si se compara con países desarrollados. Sin embargo, podemos considerarlo como relevante si se analiza el crecimiento en el consumo de leche en los últimos 25 años. Por ejemplo, en 1975 el consumo per cápita era de 1.19 litros por persona al año; en 1999 fue 7.4 litros y en 2001, 9.6 litros, de acuerdo con datos de la FAO. Esto quiere decir que de 1975 a 2001, en China se ha registrado una tasa de crecimiento promedio anual en el consumo de leche de $8.3 \%$; esto quiere decir también que en 2001 los chinos consumieron siete veces más leche que en 1975.

Otro de los aspectos que se observan en China es que, al creciente consumo interno de leche le sigue un aumento en la producción; es decir, no obstante que el crecimiento en el consumo se duplicó de 1990 a 2001 (de 8 a 16 mil millones de litros consumidos), el índice de au- tosuficiencia en leche ha crecido, pues en 1990 China producía $86 \%$ de su consumo y en 2001 produjo más de $90 \%$. El crecimiento mostrado en la producción se debió más al incremento en el hato ganadero (que fue $151 \%$ ), que a un incremento a la productividad del ganado bovino. De acuerdo con las cifras de producción y de ganado bovino, en 1990 la producción per cápita fue de 4.3 litros por día y en 2003 , de 5.6 litros por día; es decir, se registró un crecimiento en la productividad de sólo $30 \%$.

No obstante el importante crecimiento en el consumo de leche en China, hay importantes diferencias de consumo entre las distintas regiones. En las áreas urbanas, por ejemplo, los rangos de consumo oscilan de entre cero a 24 litros por persona al año, y en las áreas rurales el rango va de cero a catorce litros. En las áreas urbanas donde se registra un mayor consumo son Pekín y Shanghai; también existen regiones rurales donde se consume leche por arriba del promedio, como en las provincias lejanas de occidente (Xinjiang, Qinghai y el Tibet), regiones que son habitadas en su gran mayoría por minorías. ${ }^{11}$

Aunque los chinos cada vez están más convencidos de que consumir leche es saludable por su elevado valor nutricional, también hay creencias que se contraponen a esta idea, sobre todo en zonas rurales; por ejemplo, en

Cuadro 2

\begin{tabular}{cccc}
\multicolumn{4}{c}{ Rendimiento del ganado vacuno lechero en China } \\
\hline Año & $\begin{array}{c}\text { Total cabezas } \\
\text { (miles) }\end{array}$ & $\begin{array}{c}\text { Producción } \\
\text { (miles de litros) }\end{array}$ & $\begin{array}{c}\text { Productividad } \\
\text { (litros por dia) }\end{array}$ \\
\hline 1980 & 673 & $1,193,020$ & 4.86 \\
1985 & 1,680 & $2,589,879$ & 4.22 \\
1990 & 2,782 & $4,362,638$ & 4.30 \\
1995 & 3,967 & $6,082,213$ & 4.20 \\
2000 & 4,935 & $8,632,299$ & 4.79 \\
2003 & 7,007 & $14,335,250$ & 5.61 \\
\hline
\end{tabular}

Fuente: http://www.fao.org. 


\section{Temas varios del Pacífico}

estos lugares, la leche es considerada como un alimento caliente que debería consumirse en forma moderada (otro alimento caliente es el pollo frito). De acuerdo con la tradición, consumir en exceso estos alimentos puede provocar dolor de garganta y ampollas en la boca. En contraste, el consumo de alimentos considerados fríos, como la sandía y las peras, puede provocar orina frecuente, sudoración y diarrea.

Por lo tanto, además de los problemas mencionados arriba, esta serie de restricciones de tipo cultural limitan con frecuencia el consumo de leche en China. Incluso en las zonas urbanas, donde se está más expuesto a las ideas occidentales y los niveles de educación y de vida son más elevados, sólo $20 \%$ de las personas consumen con regularidad productos lácteos, y $30 \%$ ó $40 \%$ de los chinos que viven en estas zonas no consumen productos lácteos de ningún tipo. ${ }^{12}$

A manera de síntesis, se podría decir que las tradiciones culturales y los hábitos alimenticios no cambian tan rápidamente, incluso aunque hayan sido eliminadas algunas restricciones económicas.

\section{Política gubernamental}

El gobierno chino también ha tomado algunas decisiones que inciden en el crecimiento de la industria lechera de China. Por ejemplo, en junio de 2000, el ministro de agricultura chino publicó un ambicioso plan de largo plazo para la producción de leche. ${ }^{13}$ En ese año se producían poco más de 8 mil millones de litros de leche bovina, y se planteó el objetivo de incrementar la producción en 10 mil millones de litros más para 2005. Tomando en cuenta que la producción de leche bovina en 2003 fue de casi 14 mil millones de litros, quiere decir que el objetivo planteado en el año 2000 está tan sólo a 4 mil millones de litros más. De acuerdo con el dinamismo que ha mostrado la industria lechera y dependiendo de la fortaleza de la economía China, se espera que la producción de leche alcance una producción de entre 20 y 39 mil millones de litros en el año 2030.

Este programa contrasta con la anterior política en la que, por mucho tiempo, el gobierno apoyó el punto de vista de que el consumo de leche debía estar reservado exclusivamente para los niños, los enfermos y personas adultas. Sin embargo, en años recientes, el gobierno ha procurado incrementar no sólo la producción sino también el consumo de leche. Por ejemplo, se han lanzado campañas extensivas en los medios donde se informa a la población que es importante que incorporen a su dieta el consumo de leche, destacando la deficiencia del calcio en la mayoría de los chinos y los problemas relacionados con esta deficiencia.

Cuadro 3

Comercio de lácteos en China

(millones de dólares)

\begin{tabular}{cccc}
\multicolumn{4}{c}{ (millones de dólares) } \\
\hline Año & Importaciones & Exportaciones & Saldo \\
\hline 1990 & 307 & 13 & -294 \\
1991 & 333 & 16 & -317 \\
1992 & 343 & 22 & -321 \\
1993 & 337 & 28 & -309 \\
1994 & 350 & 24 & -326 \\
1995 & 379 & 35 & -344 \\
1996 & 372 & 33 & -339 \\
1997 & 438 & 45 & -393 \\
1998 & 337 & 47 & -290 \\
1999 & 398 & 53 & -345 \\
2000 & 438 & 62 & -376 \\
2001 & 468 & 45 & -423 \\
2002 & 456 & 61 & -395 \\
\hline
\end{tabular}

Fuente: http://www.fao.org. 
Aunque no son amplios los fondos públicos que promueven el crecimiento de la industria lechera en China, otro programa importante fue el que aplicó el ministro de agricultura con el fin de estimular el consumo de leche en las escuelas. Con este propósito, las ciudades de Pekín, Shanghai, Tianjin y Shenyang fueron seleccionadas para un programa piloto, el cual resultó ser exitoso, y se espera que contribuya de manera significativa en la demanda de leche y productos lácteos, en el mediano y largo plazo.

Según un estudio internacional realizado por Griffin y publicado en 2000, los niños que regularmente toman leche y consumen productos lácteos tienden a continuar haciéndolo como adultos, porque los hábitos alimenticios adquiridos en la niñez persisten en la edad adulta. ${ }^{14}$

Además del interés mostrado por el gobierno mediante su participación en campañas de información (donde se señalan los beneficios para la población en términos de salud) por un mayor consumo de este producto, también hay detractores que cuestionan los beneficios que se podrían obtener por un mayor consumo de leche. Por ejemplo, el Departamento de Agricultura de Estados Unidos (USDA, por sus siglas en inglés) en 1995 puntualizó que la carne y la leche no son necesarias en la ali- mentación humana. ${ }^{15}$ Otros señalan que mientras son claros los beneficios que se obtienen al consumir leche, también existen potenciales inconvenientes si se consume en demasía.

Con lo que está sucediendo en China, Derek Yach, director ejecutivo de enfermedades crónicas de la Organización Mundial de la Salud (wHo, por sus siglas en inglés), señaló que las generaciones más viejas en China se habían beneficiado con una dieta basada en un consumo alto de verduras y frutas y un consumo bajo en grasas, y que al cambiar estos hábitos alimenticios hacia un mayor consumo de leche podría deshacer estos beneficios que se habían conseguido con anterioridad. ${ }^{16}$ Finalmente, Colin Campbell, profesor de bioquímica alimenticia en Cornell y director del proyecto Cornell-China-Oxford (el más extenso jamás realizado sobre el régimen alimenticio y enfermedades), dijo que si los productos lácteos son consumidos junto con una alta dieta basada en proteína animal, cualquier potencial beneficio por el incremento en la densidad de los huesos podría ser indeterminado. Lo anterior debido a que la proteína animal, incluyendo la que proviene de los lácteos, podría extraer más calcio de los huesos, del que fuera asimilado con un mayor consumo de leche.

Este fenómeno podría explicar el hecho de que los estadounidenses que ingieren can-

\section{Cuadro anexo}

Producción de leche en China

(miles de toneladas métricas)

\begin{tabular}{cccccccccccc}
\hline Año & Total & Vaca & $\%$ & Búfala & $\%$ & Oveja & $\%$ & Cabra & $\%$ & Camella & $\%$ \\
\hline 1990 & 7,032 & 4,362 & 62 & 1,900 & 27 & 594 & 8 & 165 & 2 & 14 & 0.20 \\
1991 & 7,603 & 4,874 & 64 & 1,950 & 26 & 599 & 8 & 165 & 2 & 14 & 0.18 \\
1992 & 8,073 & 5,278 & 65 & 2,000 & 25 & 608 & 8 & 171 & 2 & 15 & 0.19 \\
1993 & 8,150 & 5,264 & 65 & 2,040 & 25 & 651 & 8 & 178 & 2 & 15 & 0.18 \\
1994 & 8,681 & 5,577 & 64 & 2,100 & 24 & 801 & 9 & 187 & 2 & 15 & 0.17 \\
1995 & 9,457 & 6,082 & 64 & 2,200 & 23 & 964 & 10 & 195 & 2 & 16 & 0.17 \\
1996 & 10,191 & 6,610 & 65 & 2,300 & 23 & 1,064 & 10 & 201 & 2 & 16 & 0.16 \\
1997 & 10,094 & 6,341 & 63 & 2,450 & 24 & 1,074 & 11 & 212 & 2 & 15 & 0.15 \\
1998 & 10,516 & 6,959 & 66 & 2,500 & 24 & 824 & 8 & 217 & 2 & 15 & 0.14 \\
1999 & 11,244 & 7,514 & 67 & 2,600 & 23 & 893 & 8 & 222 & 2 & 15 & 0.13 \\
2000 & 12,373 & 8,632 & 70 & 2,650 & 21 & 847 & 7 & 230 & 2 & 14 & 0.11 \\
2001 & 14,520 & 10,601 & 73 & 2,680 & 18 & 974 & 7 & 250 & 2 & 14 & 0.10 \\
2002 & 17,319 & 13,356 & 77 & 2,700 & 16 & 1,006 & 6 & 243 & 1 & 14 & 0.08 \\
2003 & 18,321 & 14,335 & 78 & 2,700 & 15 & 1,025 & 6 & 247 & 1 & 14 & 0.08 \\
\hline
\end{tabular}

Fuente: http://www.fao.org. 
tidades mayores de calcio tienen índices de osteoporosis y de fractura de cadera más elevados, comparado con muchos chinos y otros asiáticos que consumen pocos productos lácteos y bajos montos de calcio. Campbell dijo que las fracturas de cadera en Estados Unidos, por ejemplo, ocurren aproximadamente cinco veces más que en China. ${ }^{17}$

\section{Comercio internacional}

En términos de valor, China incrementó sus importaciones de lácteos en $48 \%$ de 1990 a 2002, al pasar de 307 a 456 millones de dólares, lo que representa una tasa de crecimiento en esos años de $3.3 \%$. Por su parte, las exportaciones también han aumentado de manera importante en porcentaje; sin embargo, en términos relativos no son muy significativas, ya que representan aproximadamente $10 \%$ del valor de las importaciones.

Para los países que son exportadores netos de productos lácteos resulta estimulante el creciente consumo de lácteos en China y el aumento de sus importaciones. Entre los principales productos lácteos importados y sus proveedores (en 1999) de leche en polvo fue Nueva Zelanda, que participó con 50\% del total; de suero, Estados Unidos con $30 \%$ del total, y finalmente $72 \%$ de la leche líquida importada provino de Australia. ${ }^{18}$

Creo que es interesante destacar que de 1990 a 2001, el crecimiento en el consumo de leche en China ha sido acompañado por un incremento ligeramente mayor en la producción, lo cual ha propiciado que el nivel de autosuficiencia en leche haya aumentado. En esos años, el consumo de leche (equivalente a litros de leche) se incrementó $96 \%$, la producción lo hizo en $105 \%$ y las importaciones $56 \%$; es decir, el aumento en la cantidad producida en términos porcentuales fue mayor que el incremento en la cantidad consumida, y en términos de la autosuficiencia alimentaria, ésta se elevó de $86 \%$ a $90 \%$.

\section{Conclusión}

China es un país que por el tamaño de su población podría representar un mercado potencial muy importante para los países que son exportadores de estos productos. Esto debido a que se han registrado niveles crecientes de consumo de lácteos, por razones de tipo económico (como los crecientes niveles de ingreso) y por campañas informativas que inducen a la población a un consumo mayor de leche. Asimismo, precisamente son estos niveles crecientes en el consumo los que podrían ocasionar un aumento importante en los precios internacionales de la leche, y esto, para el caso de países como México (que son importadores netos de leche), significa un mayor gasto y una mayor salida de divisas.

Sin embargo, así como se han incrementado el consumo y las importaciones de lácteos en China, también se observa un aumento importante en los niveles de producción, que de hecho ha permitido reducir la dependencia alimentaria de lácteos. Por esta razón, es probable que la prioridad en la política del gobierno chino se centre en el crecimiento de su industria lechera, más que pensar en una continua dependencia en sus importaciones; es decir, bajo su ambicioso plan de modernización, China probablemente priorizará sus importaciones de industrias clave como las de energía, electrónica, del transporte y de la comunicación. ${ }^{19} \mathrm{Si}$ esto es así, lo que pareciera ser un mercado potencial muy importante, tal vez no lo sea, al menos en la proporción que se esperaría dado el tamaño de su población y el creciente nivel de ingresos.

\section{Notas}

1 Michel A. Wattiaux, Gary G. Frank, J. Mark Powell, Zhiguo Wu y Yuyuan Guo. "Agriculture and dairy production systems in China: an overview and case studies". http://babcock.cals.wisc.edu/downloads/dp/ 2002-3-color.en.pdf, p. 23, años 2002-2003.

2 Op. cit., pp. 23 y 24.

3 Un importador neto de lácteos es aquel país cuyas compras por concepto de importación de lácteos son superiores a las ventas por concepto de exportación de lácteos. 
4 http://www.fao.org.

5 Se incluyen también las importaciones.

6 Robert Goodland. "The westernization of diets: the assessment of impacts in developing countries- with special referente to China. http://www.globalhunger.net/ GoodlandChina.pdf, p. 22, año 2001.

7 Michel A. Wattiaux, op. cit., p. 25.

8 Hengyun Ma y Allan Rae. "Projections of dairy product consumption and trade opportunities in China". http:// econ.massey.ac nz/caps/dairy_aust_agribus.pdf, consulta, 18 de marzo de 2004 pp. 2 y 15.

9 Stanley Fisher, Rudiger Dornbusch y Richard Schmalensee (1996) Economía. México: McGraw-Hill.

10 Elasticidad renta de la demanda es la variación porcentual que experimenta la cantidad demandada cuando aumenta la renta un $1 \%$. Un bien de lujo tiene una elasticidad renta de la demanda mayor que 1 , un bien necesario tiene una elasticidad renta de la demanda menor que 1. Si la elasticidad renta de la demanda es mayor que 1 , un aumento de la renta de un $1 \%$ eleva la cantidad demandada más de un $1 \%$, manteniéndose todo lo demás constante.

11 Michel A. Wattiaux, op. cit., p. 25.

12 Ibidem, p. 26

13 Idem.

14 Michel A. Wattiaux, op. cit., p. 27.

15 Robert Goodland, op. cit., p. 3.

16 Kathy Chen. "Got milk? The new craze in China is dairy drinks". http://www.mindfully.org/Food/2003/ChinaDairy-Drinks28feb03.htm, año 2003.

$17 \mathrm{http}: / /$ www.news.cornell.edu.

18 Michel A. Wattiaux, op. cit., p. 28.

19 Idem. Min 